\title{
Helicobacter pylori infection and inflammatory bowel disease: a crosstalk between upper and lower digestive tract
}

\author{
Yang $\mathrm{Yu}^{1}$, Shengtao Zhu', Peng Li', Li Min (1) and Shutian Zhang (1)
}

\begin{abstract}
Helicobacter pylori has coexisted with humans for approximately 60,000 years and greater than $50 \%$ of the global population is infected with H. pylori. H. pylori was successfully cultured in vitro in 1983 and studies of H. pylori have achieved substantial advances over the last 35 years. Since then, $H$. pylori has been characterized as the primary pathogenic factor for chronic gastritis, peptic ulcer, and gastric malignancy. Numerous patients have received H. pylori eradication treatment, but only $1-2 \%$ of $\mathrm{H}$. pylori-infected individuals ultimately develop gastric cancer. Recently, numerous epidemiological and basic experimental studies suggested a role for chronic H. pylori infection in protecting against inflammatory bowel disease (IBD) by inducing systematic immune tolerance and suppressing inflammatory responses. Here we summarize the current research progress on the association between $\mathrm{H}$. pylori and IBD, and further describe the detailed molecular mechanism underlying $H$. pylori-induced dendritic cells (DCs) with the tolerogenic phenotype and immunosuppressive regulatory T cells (Tregs). Based on the potential protective role of $H$. pylori infection on IBD, we suggest that the interaction between $\mathrm{H}$. pylori and the host is complicated, and $\mathrm{H}$. pylori eradication treatment should be administered with caution, especially for children and young adults.
\end{abstract}

\section{Facts}

- IBD etiology is mainly attributed to the complex interaction between immune dysfunction, host genetic susceptibility, and environmental factors.

- Epidemiological and basic experimental studies both suggested a protective role of chronic $H$. pylori infection against IBD.

- This protective effect on IBD could be attributed to H. pylori-induced systematic immune tolerance and the suppression of inflammatory response.

- Tolerogenic phenotype DCs and immunosuppressive Treg are thought to be involved

Correspondence: Li Min (minli@ccmu.edu.cn) or

Shutian Zhang (zhangshutian@ccmu.edu.cn)

'Department of Gastroenterology, Beijing Friendship Hospital, Capital Medical University, National Clinical Research Center for Digestive Disease, Beijing

Digestive Disease Center, Beijing Key Laboratory for Precancerous Lesion of Digestive Disease, Beijing 100050, China

Edited by G. HÃcker in the protective mechanisms.

- Low bioactive LPS of $H$. pylori could not effectively activate NF- $\mathrm{KB}$ pathway and stimulate the secretion of proinflammatory factors. IL-10, TGF- $\beta$, NLRP3 inflammasome, and IL-18 are critical for the protective effect of $H$. pylori on IBD.

\section{Open questions}

- Multicenter cohort studies revealing the status of $H$. pylori infection immediately after diagnosis of IBD is highly desirable.

- Prospective studies focusing on the pathogenesis or progression of IBD after $H$. pylori eradication therapy is urgently needed.

- The relationship between enterohepatic helicobacteria species and IBD needs to be further revealed.

- The detailed molecular mechanism underlying $H$.

\section{() The Author(s) 2018}

(c) (i) Open Access This article is licensed under a Creative Commons Attribution 4.0 International License, which permits use, sharing, adaptation, distribution and reproduction in any medium or format, as long as you give appropriate credit to the original author(s) and the source, provide a link to the Creative Commons license, and indicate if changes were made. The images or other third party material in this article are included in the article's Creative Commons license, unless indicated otherwise in a credit line to the material. If material is not included in the article's Creative Commons license and your intended use is not permitted by statutory regulation or exceeds the permitted use, you will need to obtain permission directly from the copyright holder. To view a copy of this license, visit http://creativecommons.org/licenses/by/4.0/. 
pylori-induced tolerogenic phenotype DCs and immunosuppressive Tregs is not yet clear.

- Considering the trade-off between gastric cancer prevention and the risk of triggering of IBD, whether an asymptomatic $H$. pylori infection should be provided with an eradication prescription is still worth discussing.

\section{Introduction}

Inflammatory bowel disease (IBD) is characterized by chronic, nonspecific intestinal inflammation with an unexplained pathology and an alternating relapsing and remitting clinical progression. IBD is divided into two subtypes: ulcerative colitis (UC) and Crohn's disease (CD). The pathological features of IBD include enhanced TH1 and/or TH17 responses, and dramatically increased production of inflammatory factors in mucosal lesions, including tumor necrosis factor- $\alpha$, interleukin (IL)- $1 \beta$, interferom (IFN)- $\gamma$, IL-17, IL-6, and IL-23 ${ }^{1-7}$. Most studies in the IBD field attribute its etiology to the complex interactions among immune dysfunction, genetic susceptibility of the host, and environmental risk factors. Autoimmune abnormalities are now widely considered one of the causes of IBD. Most patients with IBD have an individual or family history of nodular erythema, arthritis, ophthalmic uveitis, vasculitis, or systemic lupus erythematosus. In addition, mutants in autophagy genes (ATG16L1/NOD2/IRGM) were identified as inducers of aberrant immunopathological responses and impair the mucosal barrier ${ }^{8,9}$. In addition, the intestinal flora is considered an indispensable factor for intestinal inflammation, as most germ-free IL-10-deficient mice never develop colitis ${ }^{10-12}$. Given the dramatically increased prevalence in most developing countries ${ }^{9,13-20}$, IBD has become a substantial global medical burden and modern refractory disease, as cited by the World Health Organization, in the last two decades ${ }^{1,13-15}$.

Helicobacter pylori is a Gram-negative, spiral-shaped bacillus. It successfully colonizes the gastric mucosa due to its specific motility, microaerobic metabolism, and antiacid activity ${ }^{21}$. H. pylori secretes vacuolating cytotoxin (VacA) and cytotoxin associated gene A antigen(CagA) proteins and other virulence factors to induce a TH1dominated inflammatory response. Although Warren and Marshall $^{22}$ first discoverd H. pylori in 1983, H. pylori has coexisted with humans for a considerably longer period. Biogeography studies cite $H$. pylori as a witness to human migration history from East Africa approximately 60,000 years ago, and paleomicrobiologists found $H$. pylori in the oldest mummies and the Alps Iceman who lived in 5200 years ago. In the early twentieth century, numerous researchers observed the spiral bacteria on gastric mucosal surface, but the existence of $H$. pylori was not confirmed until Warren and Marshall ${ }^{22}$ discovered it.
Subsequently, researchers found almost all $H$. pyloriinfected patients exhibit histological, chronic, active inflammation, even asymptomatic $H$. pylori-infected individuals $^{23}$. In addition, the inflammatory response is reduced after $H$. pylori eradication and $H$. pylori was identified as the pathogenic factor that directly causes chronic gastritis and a class I biological carcinogenic factor in gastric cancer ${ }^{24}$. According to the 2015 "Helicobacter pylori gastritis Kyoto global consensus report", $H$. pylori gastritis should be defined as an "infectious disease" and all $H$. pylori-positive patients should receive eradication therapy, regardless of the presence of gastric ulcers or gastric cancer ${ }^{25}$. However, although approximately half of the global population is infected with $H$. pylori, only $10-20 \%$ of $H$. pylori-infected individuals exhibit peptic ulcers, 1 2\% develop gastric cancer, and < $1 \%$ exhibit gastric mucosa-associated lymphoid tissue lymphoma ${ }^{26-28}$. Moreover, consistent with "Africa enigma," recently reported gastric cancer prevalence is also much lower in less developed Asian countries (who have high $H$. pylori infection rates range of 55-92\%) than relatively developed Asian country ${ }^{29}$.

\section{Association between $\boldsymbol{H}$. pylori and IBD}

Recently, emerging epidemiologic studies and animal experiments $^{30}$ revealed an inverse correlation between $H$. pylori infection and IBD onset, suggesting that $H$. pylori colonization exerts a special protective effect on autoimmune diseases. Since the twenty-first century, improving hygienic conditions and socioeconomic status have reduced the $H$. pylori infection rate and this trend has concurrently been accompanied by an increased IBD incidence in most countries ${ }^{31}$. Most experts in the IBD field interpret this phenomenon based on the "hygiene hypothesis": H. pylori infection during childhood contributes to immune system development and may prevent the onset of autoimmune or allergic diseases. Moreover, due to the initiation of $H$. pylori eradication for peptic ulcers, the incidence of IBD has increased steadily in these regions ${ }^{9}$. Further clarification of the protective effect of $H$. pylori on IBD and the underlying mechanism will be important for $H$. pylori infection management strategies and the treatment and prevention of IBD (Fig. 1 and Fig. 2).

\section{Enterohepatic helicobacteria participate in IBD pathogenesis}

Various non-pylori Helicobacter organisms in the Helicobacteraceae family have been found to be able to colonize throughout the gastrointestinal tract and are defined as enterohepatic helicobacteria species (EHS). In addition, 16s rDNA sequencing of colonic biopsies ${ }^{32,33}$ and fecal samples ${ }^{34,35}$ revealed an increased prevalence of the Helicobacteraceae family in children with $C D$, 


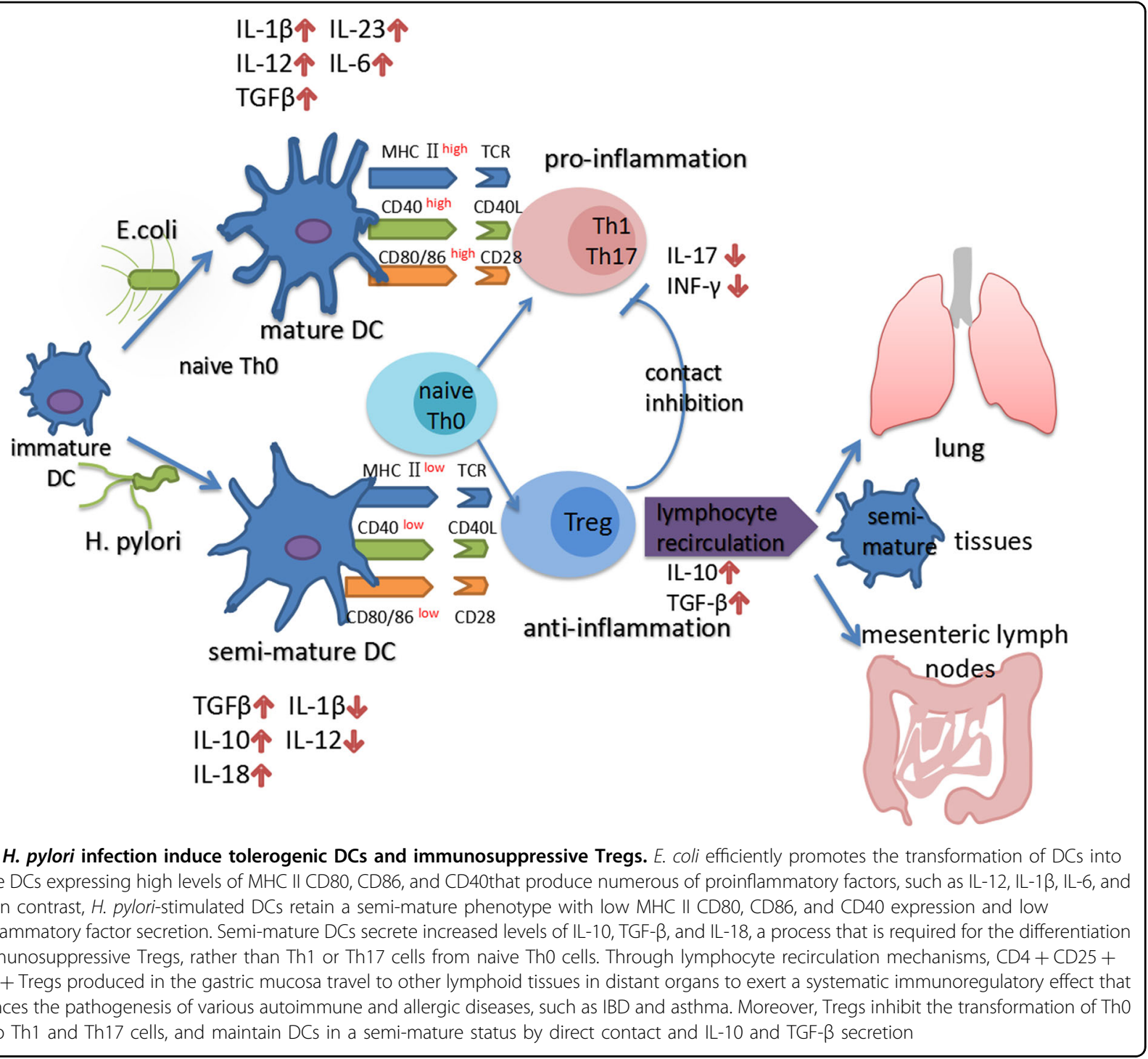

particularly Helicobacter bilis and Helicobacter hepaticus. A meta-analysis ${ }^{36}$ further revealed an increased prevalence of EHS among patients with IBD compared with the control group ( $R R($ relative risk) $=2.01,95 \%$ confidence interval (CI): 1.36-2.98). In fact, some EHS have been routinely used to induce experimental colitis in immunodeficient animals ${ }^{37}$. In a study on the pathogenic mechanism of EHS by Kullberg et al. ${ }^{38}$., H. hepaticus infection elicited persistent colitis in IL- $10^{-1-}$ mice by stimulating an IL-12(p35/P40)-dependent Th1 reaction. Subsequently, Kullberg et al. ${ }^{39}$. further verified that the IL-23(P40/P19)-dependent Th17 reaction also played a key role in an $H$. hepaticus-induced mouse colitis model. Other pathogenic mechanisms reported in related studies include disruption of the intestinal epithelial integrity by the type VI secretion system ${ }^{40}$, disruption of the eukaryotic cell cycle via the production of a cytolytic distending toxin $^{41}$, and alterations in normal flora colonization to reduce flora diversity ${ }^{42,43}$. Based on these findings, intestinal Helicobacteraceae colonization is a potentially pathogenic factor for IBD, not a protective factor.

The potential protective effect of $\boldsymbol{H}$. pylori infection on IBD

Numerous studies have reported a lower $H$. pylori infection rate in patients with $\mathrm{CD}$ and/or UC than in nonIBD control individuals ${ }^{44,46-49,51-55,57-66}$, although a small number of studies showed no significant association ${ }^{45,50,56}$ (Table 1). The inverse correlation between IBD and $H$. pylori infection suggests that the gastric mucosa colonization of $H$. pylori can potentially protects against the pathogenesis of IBD via a special mechanism. Two metaanalyses $^{67,68}$ (including 23 and 33 studies, separately) provide more powerful evidence supporting this protective effect of $H$. pylori infection on the prevalence of IBD 


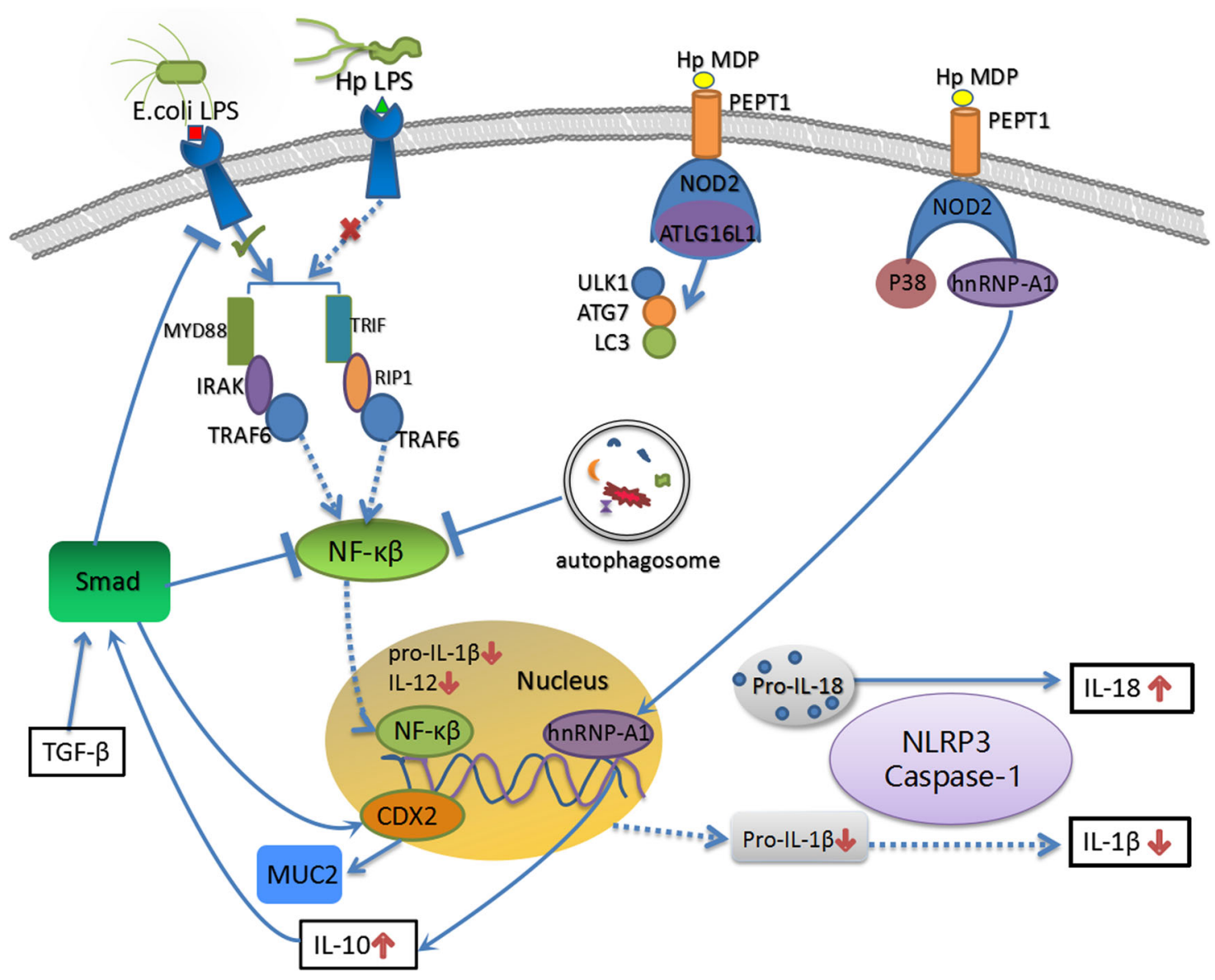

Fig. 2 The molecular mechanism underlying the protective effect of $\boldsymbol{H}$. pylori on IBD. The uncommon structure and weak biological activation of $H$. pylori LPS leads to the inefficient activation of NF-KB and production of low levels of proinflammatory molecules. On the other hand, $H$. pylori activates NOD2 and ATG16L1 to activate autophagy, and the process of autophagosome formation results in the endocytosis of MHC II and inhibition of NF-KB. The disequilibrium between inflammation and autophagy (the latter is relatively enhanced by $H$. pylori infection) may have a key role in the formation of tolerogenic semi-mature DCs. Moreover, NOD2 forms trimers with p38 and hnRNP-A1, and the latter subsequently enters the nucleus to stimulate IL-10 transcription. IL-10 and TGF- $\beta$ are required for the activation of the Smad signaling pathway and downstream protective mechanisms, including the inhibition of TLR expression and the NK-KB signaling pathway and the induction of CDX2 production and MUC2 transcription. In addition, NLRP3 and IL-18 are indispensable for the protective effect of H. pylori on experimental colitis. Due to the NF-KBindependent production mechanism, pro-IL-18 is stably expressed in the cytoplasm and is effectively produced by activated NLRP3 and caspase-1 after $H$. pylori infection

$(\mathrm{RR}=0.64,95 \% \mathrm{CI}: 0.54-0.75$ and $\mathrm{RR}=0.62,95 \% \mathrm{CI}:$ $0.55-0.71)$. However, the significant heterogeneity ${ }^{67,68}$ among the included studies and the potential publication bias $^{68}$ largely limited the confidence of this negative correlation. Differences in H. pylori detection methods, IBD diagnostic criteria, study sites, participant ages, and histories of antibiotic therapy potentially contribute to the severe heterogeneity, which was not resolved by a subgroup analysis. However, a recent meta-analysis ${ }^{69}$ without statistical heterogeneity and publication bias also reported an inverse correlation ( $\mathrm{RR}=0.48,95 \% \mathrm{CI}$ : 0.43-0.54) between $H$. pylori infection and IBD prevalence in an Asian population (Table 2).

Some researchers ${ }^{70-73}$ attributed this inverse correlation to the complex medical therapies used by patients with IBD, including metronidazole, quinolone drugs, sulfasalazine, 5-aminosalicylic acid, corticosteroids, and immunosuppressants. The intake of these medications was considered a possible cause of the "spontaneous eradication" effect that leads to the low $H$. pylori infection rate in patients with IBD. However, this conclusion was not supported by other studies ${ }^{53,54,57,74,75}$, which reported that a history of taking sulfasalazine, 5-aminosalicylic acid, corticosteroids, and immunosuppressants was not a confounding factor for this inverse correlation. In addition, even if antibiotics reduce $H$. pylori infection rates in patients with IBD, the $H$. pylori infection rate remains significantly reduced in patients with IBD without a history of antibiotics use compared with healthy controls ${ }^{53,54,57,74,75}$. Multicenter prospective cohort studies 


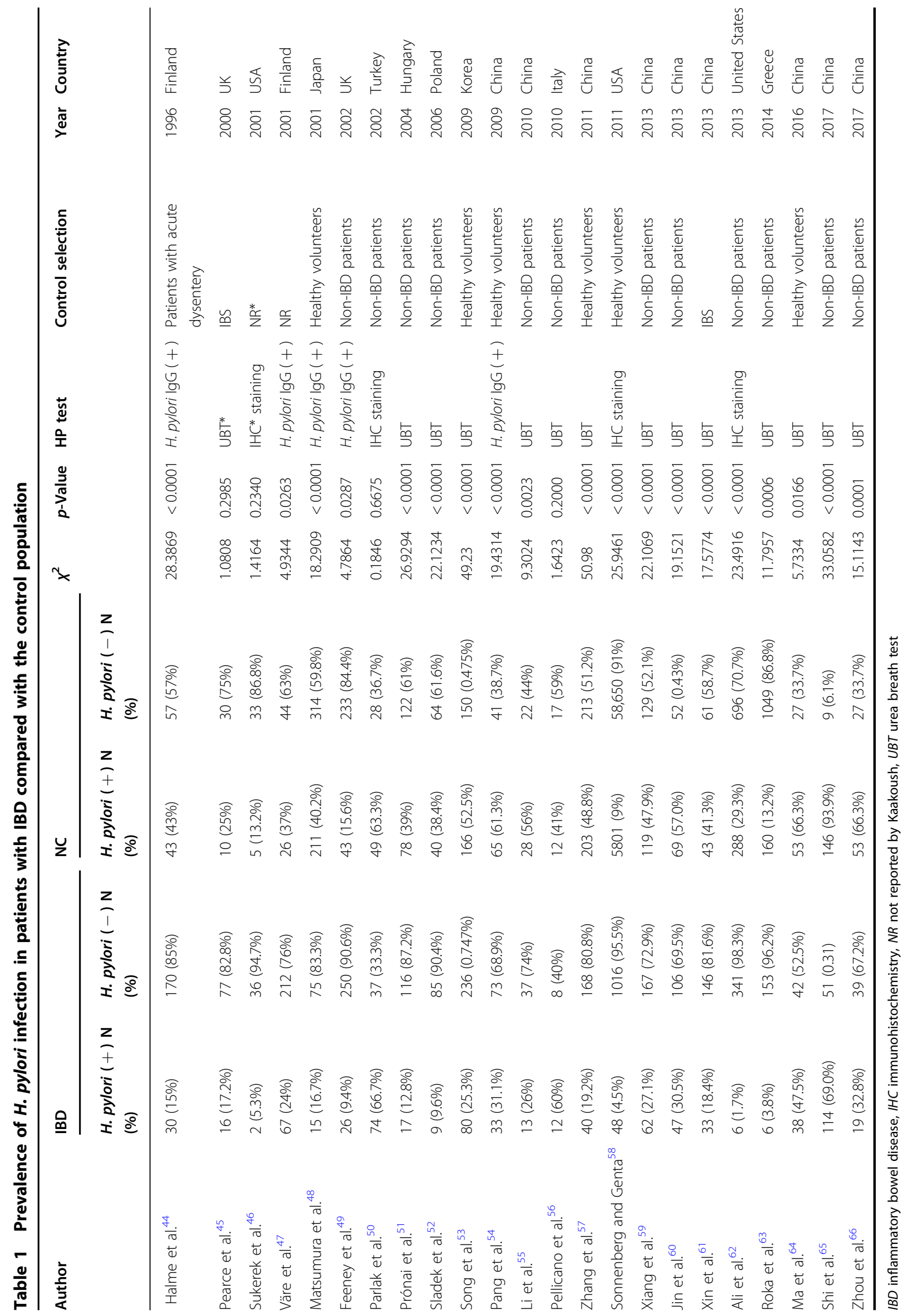


Table 2 Meta-analysis of $H$. pylori infection rates in patients with IBD

\begin{tabular}{|c|c|c|c|c|c|c|c|}
\hline \multirow[t]{2}{*}{ Author } & \multirow[t]{2}{*}{ Subgroup } & \multirow[t]{2}{*}{ Pooled RR/OR } & \multirow[t]{2}{*}{$95 \% \mathrm{Cl}$} & \multirow[t]{2}{*}{$p$-Value } & \multicolumn{2}{|c|}{ Heterogeneity } & \multirow[t]{2}{*}{ Publication bias } \\
\hline & & & & & $I^{2}$ & $p$-Value & \\
\hline \multirow[t]{3}{*}{ Luther et al. ${ }^{67}$} & IBD & 0.64 & $0.54-0.75$ & NR & $75.80 \%$ & $<0.001$ & NR \\
\hline & $C D$ & 0.6 & $0.40-0.72$ & NR & NR & NR & \\
\hline & UC & 0.75 & $0.62-0.90$ & NR & NR & NR & \\
\hline \multirow[t]{3}{*}{ Rokkas et al. ${ }^{68}$} & $\mathrm{IBD}$ & 0.62 & $0.55-0.71$ & $<0.001$ & $77 \%$ & $<0.001$ & 0.15 \\
\hline & $C D$ & 0.38 & $0.31-0.47$ & $<0.001$ & $59.50 \%$ & $<0.001$ & \\
\hline & UC & 0.53 & $0.42-0.67$ & $<0.001$ & $62 \%$ & $<0.001$ & \\
\hline \multirow[t]{3}{*}{ Wu et al. ${ }^{69}$} & $\mathrm{IBD}$ & 0.48 & $0.43-0.54$ & $<0.001$ & $21 \%$ & $N R$ & 0.203 \\
\hline & $C D$ & 0.43 & $0.37-0.50$ & $<0.001$ & $43.00 \%$ & $N R$ & \\
\hline & UC & 0.55 & $0.48-0.64$ & $<0.001$ & $0 \%$ & NR & \\
\hline \multirow[t]{3}{*}{ Castañorodríguez et al. ${ }^{74}$} & $\mathrm{IBD}$ & 0.426 & $0.362-0.502$ & $<0.001$ & $62 \%$ & $<0.001$ & NR \\
\hline & $C D$ & 0.38 & $0.31-0.47$ & $<0.001$ & $N R$ & NR & \\
\hline & UC & 0.53 & $0.44-0.65$ & $<0.001$ & NR & NR & \\
\hline
\end{tabular}

$C D$ Crohn's disease, $C I$ confidence interval, IBD inflammatory bowel disease, $O R$ odds ratio, UC ulcerative colitis

that confirm the H. pylori infection status and therapy history immediately after IBD diagnosis are urgently needed, and better control of confounding factors in these studies should be implemented to achieve definitive conclusions.

Animal experiments also confirmed the negative correlation between $H$. pylori infection and IBD onset. As shown in the study by Fen et al. ${ }^{76}, H$. pylori infection significantly ameliorates colitis and histopathological changes in a DSS-induced mouse colitis model. This pathological difference is accompanied by reductions in splenic CD4 + T cells and the extent of systemic inflammation. Using mice co-infected with $H$. pylori and Salmonella typhimurium, Higgins $\mathrm{PD}^{77}$ reported that $H$. pylori inhibits the Th17 response to S. typhimurium infection and increases IL-10 levels in mesenteric lymph nodes. Based on the results of these studies, $H$. pylori infection affects the immune response in the lower digestive tract and involves potential immunological crosstalk between the upper and lower gastrointestinal tracts.

\section{$H$. pylori infection induces tolerogenic DCs Dendritic cells (DCs) capture $H$. pylori antigens in the gastric cavity}

Although numerous epidemiological studies and metaanalyses support the inverse correlation between $H$. pylori infection and IBD onset, the protective mechanism by which the upper digestive tract colonization of $H$. pylori can protect against IBD remains unclear. As the most powerful antigen-presenting cell and the unique activator of naive T lymphocytes (Th0), DCs have a key role in modulating adaptive immunity through the presentation of pathogen antigens and induce Th0 cells to differentiate into different lymphocyte subsets. Using two-photon microscopy to observe transgenic pCD11c-YFP mice, Kao et al. ${ }^{78}$ reported that $\mathrm{CD} 11 \mathrm{c}+\mathrm{DCs}$ are located near the gastric luminal surface and submucosal layer, and the number of DCs in the lamina propria was dramatically increased and DCs moved closer to the epithelial surface after $H$. pylori infection. Moreover, through a threedimensional co-culture system that includes monocytes, DCs and a Caco- 2 cell monolayer in a type I bovine collagen system, Leonard et al. ${ }^{79}$ observed DCs can move to the surface of Caco-2 cell monolayer or integrated with it. These studies indicated DCs can migrate through the intestinal epithelium to sense gastrointestinal tract antigens without impairing the integrity of the epithelial barrier.

\section{H. pylori remodel DCs to exhibit an immune tolerance property}

Investigations focused on the tolerogenic property of $H$. pylori-specific DCs may help reveal the intriguing mechanism by which $H$. pylori induces systematic immunosuppression. Oertli et al. $^{80}$ purified gastric mucosa lamina propria-derived DCs from $H$. pyloriinfected patients and found that these DCs express high levels of HLA-DR and SIGN but low levels of CD80, $\mathrm{CD} 83$, and CD86. Kao et al. ${ }^{78}$ further studied the different cytokines secreted by bone marrow-derived DCs after stimulation with $H$. pylori, Escherichia coli, and Ruffey's 
Acinetobacter. In this study, H. pylori-stimulated DCs not only maintained high transforming growth factor (TGF) $-\beta$ levels but also displayed lower levels of IL- 6 and IL-23 expression level than DCs stimulated with the other two positive control bacteria. IL-6 and IL-23 are important inflammatory factors that have key roles in Th17 differentiation and function maintenance ${ }^{6,81-84}$, suggesting that H. pylori has a poor pathogenicity that cannot effectively activate the inflammation pathway and Th17-modulated proinflammatory responses. This tolerogenic property also has been observed at the level of DC surface molecules. In the study by Oertli et al. ${ }^{80}$, prestimulate DCs with $H$. pylori in vitro significantly suppressed the E. coli lipopolysaccharide (LPS)-induced upregulation of CD80, CD86, and CD40. In addition, significantly lower IL-12 p40 and IL-6 levels were observed in H. pylori-prestimulated DCs than in the E. coli LPS-treated group (summarized schematically in Figure 1). Based on these evidence, although $H$. pylori infection recruits numerous DCs to the gastric mucosa, these DCs exhibit a functionally semi-mature status with an immune tolerance phenotype. This immune tolerance property of $H$. pylori may contribute to its persistent colonization of the gastric mucosa and its ability to simultaneously exert a systematic immunomodulatory effect to suppress autoimmune immunopathological responses.

\section{Molecular mechanism by which $H$. pylori induces tolerogenic DCs}

The intrinsic nature of immune tolerance induced by $H$. pylori is attributed to the low bioactivity of its LPS. By administering intravenous injections of different LPS doses and performing three typical in vitro endotoxin tests, Muotiala et al. ${ }^{85}$ observed an approximately 500 - to 1000-fold reduction in the biological activation of $H$. pylori LPS compared with two Salmonella enterica serovar Typhimurium subspecies (Figure 2). Long 3-hydroxy fatty acids and a deficiency of phosphorylated groups at position 4' in the D-glucosamine disaccharide backbone of Lipid A, a constituent component of LPS, potentially explain the reduced biological activity. This uncommon structure and the significantly weaker biological activation of $H$. pylori LPS may be responsible for the formation of tolerogenic semi-mature DCs. In addition, modifications in the N-terminal TLR5 recognition domain of $H$. pylori flagellin may contribute to the escapes recognition by TLR5 (Figure 2) ${ }^{86}$. H. pylori induces DC proliferation and activates autophagosome formation in vitro ${ }^{87}$. H. pylori infection-induced autophagy activity may participate in DC remodeling process; LC3, LAMP1, and major histocompatibility complex (MHC) class II molecules were found retained in autophagic vacuoles after $H$. pylori infection; meanwhile, the surface expression of MHC II, CD80, and CD86 decreases in a TLR2/TLR4-dependent manner. Moreover, no IL-12 was detected in DCs stimulated with wild-type or VacA/CagA mutant $H$. pylori strains consistent with the downregulation of DC function and impaired T-cell proliferation (Figure 2). Based on these results, $H$. pylori infection induces TLR2/TLR4dependent autophagy to downregulate DC function and inhibit T-cell proliferation. However, the detailed mechanism by which $H$. pylori participates in the interaction between autophagy activation and inflammatory pathways remains to be further elucidated. Moreover, some virulence factors may be necessary for the protective effect of $H$. pylori on IBD and asthma ${ }^{88}$. Lord et al. ${ }^{89}$ reported a significantly lower CagA-positive rate in patients with $\mathrm{CD}(0.94 \%)$ than in unaffected individuals (7.48\%), suggesting that the CagA protein may participate in the IBD protective mechanism. Oertli et al. ${ }^{90}$ and Engler et al. ${ }^{91}$ demonstrated two dominant virulence factor $\gamma$-glutamyl transpeptidase and VacA were essential for $H$. pylori-induced tolerogenic re-programming of DCs in vivo and in vitro asthma model. However, contradictory conclusions were obtained from colitis animal $\operatorname{model}^{78,80}$; in these studies, the immunomodulatory effect of $H$. pylori-stimulated DCs was independent of VacA or CagA.

\section{H. pylori infection induces immunosuppressive Tregs Tregs participate in $\mathrm{H}$. pylori-induced immune tolerance}

As $H$. pylori strictly colonizes the gastric mucosa, the mechanism by which $H$. pylori remotely modulates lower digestive tract immune responses to influence the pathogenesis of IBD is still a subject of debate. Recently, emerging animal and in vitro experiments provided thought-provoking evidence that $H$. pylori infection of the upper digestive tract can modulate the systemic immune response by remodeling DCs to exhibit immune tolerance properties and subsequently induce Tregs polarization. Tregs are one lymphocyte subgroup that suppresses the activity of effector $\mathrm{T}$ cells and has a key role in maintaining immune system homeostasis and self-tolerance ${ }^{92}$. Forkhead box transcription factor (FOXP3) expression is required for this immunosuppressive function of Tregs. Foxp3-expressing regulatory $\mathrm{B}$ cell can upregulate Treg/Th17 ratio to ameliorate autoimmune arthritis $^{93}$. Foxp3-knockout mice develop various severe or even fatal metabolic, allergic, and autoimmune diseases ${ }^{94-97}$. Tregs can suppress effector $\mathrm{T}$-cell differentiation and proliferation by direct contact inhibition or anti-inflammatory cytokine secretion. Moreover, Tregs was shown can diminish the upregulation of costimulatory molecule on splenic $\mathrm{DCs}^{98}$. Tregs also participate in the pathogenesis of $H$. pyloriinduced chronic gastritis and many studies report increased numbers of CD $4+$ CD25 + Foxp $3+$ Tregs in 
the gastric mucosa of patients with $H$. pylori infections ${ }^{99,100}$. Transfer of Tregs derived from H. pyloripretreated neonatal mice donor attenuated ovalbumininduced allergic airway inflammation when compared with challenged control mice ${ }^{88}$. Conversely, systemic Treg depletion abolished this protection effect ${ }^{101}$. More evidence was reported by Kao et al. ${ }^{78}$; they stimulated MACS(Magnetic Activated Cell Sorting) microbeadisolated splenic $\mathrm{CD} 4+\mathrm{T}$ cells with bone marrowderived DCs and $H$. pylori SS1 in vitro, and found that $H$. pylori induces an increased Treg ratio and decreases IL-17 levels in an IL-10- and TGF- $\beta$-dependent manner. Moreover, adoptive transfer of $H$. pylori SS1-stimulated DCs in mice induces a peripheral H. pylori-specific Treg response that is characterized by increased IL-10 secretion from splenic CD4 $+\mathrm{T}$ cells. Thus, H. pyloristimulated DCs can subsequently promote Treg differentiation to induce immune tolerance.

\section{Tregs have a key role in systematic immunomodulation}

As Tregs are required to prevent dysfunctional inflammatory responses to commensal organisms in the lower digestive tract ${ }^{102}$, Tregs may have a central role in chronic H. pylori infection-induced systematic immunomodulation and exert protective effects on IBD. This hypothesis was further verified by the effectiveness of Treg adoptive transfer therapy on mouse models of colitis or asthma ${ }^{103,104}$. In contrast, the dramatically reduced $H$. pylori colonization density after Treg depletion was accompanied by an enhanced peripheral Th17 response. In addition, $H$. pylori-positive patients typically present with lower peripheral type I IFN levels than the control group ${ }^{105}$. Based on the lymphocyte recirculation theory, we proposed that although $H$. pylori strictly colonizes the gastric mucosa, $H$. pylori-induced Tregs arrive at remote organs to suppress effector T-cell proliferation and elicit a systematic immunoregulatory effect(Figure 1). Furthermore, Onishi et al. ${ }^{106}$ found Tregs can aggregate around DCs and subsequently downregulate the costimulatory molecules CD80 and CD86 to maintain the semi-mature phenotype of DCs (Figure 1). Together with the lymphocyte recirculation theory, these findings explain the increase in the lung infiltration of semi-mature DCs in $H$. pylori-infected mice $^{101}$, as $H$. pylori is unlikely to directly influence the respiratory system. In conclusion, the considerable number of Tregs induced by persistent H. pylori colonization in the upper digestive tract may exert a systematic immunoregulatory effect on remote organs via lymphocyte recirculation and might ultimately influence the pathogenesis of various autoimmune and allergic diseases, such as IBD and asthma.

\section{Molecular mechanism by which $H$. pylori induces immunosuppressive Tregs}

TGF- $\beta$ and IL-10 are two important and wellrecognized immunoregulatory factors ${ }^{107,108}$, and these molecules are associated with IBD onset and Tregmodulated intestinal mucosal homeostasis ${ }^{109-111}$, which suggest that tolerogenic DCs may induce and maintain Treg differentiation via IL-10- and TGF- $\beta$-dependent mechanisms. Pretreatments with TGF- $\beta$ - and IL-10neutralizing antibodies reversed the ameliorated colitis pathology and the upregulation of Treg/Th17 ratio after H. pylori stimulation further proved this hypothesis ${ }^{12,113}$. Intestinal epithelial cells derived from IL-10 ${ }^{-1-}$ mice only express RelA (p65, a phosphorylated nulcear factor (NF)$\kappa B$ subunit), but not phosphorylated Smads, after pathogen stimulation ${ }^{114}$. Meanwhile, TGF- $\beta$ was shown to activate Smad signaling to inhibit Toll-like receptor (TLR) expression and NF- $\mathrm{kB}$ pathway-related proinflammatory cytokine secretion ${ }^{115}$. In addition, Engler et al. ${ }^{116}$ revealed a significant correlation among CDX2, MUC2, and TGF$\beta$, and demonstrated the activation of the TGF- $\beta$ dependent Samd-CDX2-MUC2 axis after $H$. pylori infection or extraction treatment can increases intestinal mucus secretion and ameliorate experimental colitis (summarized schematically in Figure 2). In summary, these evidences indicated TGF- $\beta$ and IL-10 are critical factors for Treg differentiation and activation of protective Smad signaling after bacterial pathogen stimulation.

H. pylori can be successfully sensed by TLR2/NOD2 and subsequently activate NLRP3 inflammasome and caspase- 1 to promote the maturation of IL- $1 \beta$ and IL$18^{117,118}$. The essential role of NLRP3 inflammasome and IL-18 for the protective effect of $H$. pylori on experimental colitis was proved by Engler et al. ${ }^{116}$. They found $\mathrm{Nlrp} 3^{-/-}$, IL-18 ${ }^{-/-}$, and IL18R ${ }^{-/-}$deficient mice all lack the effective protective effect of a live $H$. pylori oral infection or intraperitoneal injection of extracts. Moreover, IL-18 was found to be required for Treg differentiation in vivo and in vitro ${ }^{80}$. LPS was previously shown to activate NF- $\mathrm{KB}$ pathway and significantly promote proIL-1 $\beta$ transcription to induce Th17 differentiation and stimulate powerful inflammatory response ${ }^{119,120}$. Although LPS also induces pro-IL-18 processing via the NLRP3 inflammasome, this process occurs independently of NF- $\mathrm{KB}$ activation due to stable storage of pro-IL-18 in cytoplasmic granules. Therefore, the inefficient perception by TLR4 and diminished NF-кB pathway due to low activity of $H$. pylori LPS lead to decreased pro-IL- $1 \beta$ and IL-1 $\beta$ levels, but not for IL-18 expression (summarized schematically in Figure 2). As IL-1 $\beta$ has been shown to be a strong proinflammatory cytokine ${ }^{121,122}$, the alterations in the relative expression levels of IL-1 $\beta$ and IL-18 may strikingly skew the Th1/Th17-dominated 
proinflammatory response to a Treg-dominated immunosuppressive response.

\section{The crosstalk between HP eradication and the immune response}

Although $H$. pylori-associated gastroenteritis is characterized by the aggregation of local lymphocytes and polymorphonuclear cells, $H$. pylori can persistently colonize the gastric mucosa, depending on its immune escape mechanism. According to previous studies ${ }^{123,124}$, relatively mild gastritis in children is typically accompanied by higher levels of the Foxp3 mRNA and regulatory cytokine (IL-10 and TGF- $\beta$ ) expression, as well as decreased levels of the IL-17 mRNA and neutrophil infiltration in the gastric mucosa than adults with more severe gastritis. Neonatally infected mice exhibit higher density of $H$. pylori colonization due to the lack of CD4 + T-cell infiltration into the gastric mucosa. Meanwhile, neonatally infected mice derived DCs incompetently inducing Th1 effector responses from naive $\mathrm{T}$ cells than adult-infected group $^{80}$. Futhermore, in a DSS-induced colitis mouse model, mice infected during neonatal period showed less pathology and less proinflammatory cytokine secretion ${ }^{125}$. These finding can be attributed to the different pathogenicity sense ablility and CD4 $+\mathrm{T}$-cells differentiation tendency between children and adult ${ }^{126}$. Above evidences indicate young people whose immune system may still get remodeled can benefit more from the immune tolerance induced by $H$. pylori than older people. Another intriguing phenomenon is the significantly higher success rate of $H$. pylori clearance in patients with ulcers compared with patients with chronic gastritis. A reasonable interpretation is the immune tolerogenic property of $H$. pylori, which acquired in the long co-evolution history with human, can polarize adaptive immune to Foxp3 + Tregdominated immunoregulatory response to favor its persistent colonization. Given the large number of Tregs and their immunosuppressive properties, patients with chronic gastritis cannot elicit a sufficiently effective immune response to eradicate $H$. pylori. However, in patients with ulcers, the breakdown of the balance between Tregs and Th1/Th17 cells transform the immune system to the latter dominating proinflammatory response, leading to more severe pathological lesions. Meanwhile, $H$. pylori is easier to eradicate using exogenous antibiotic and proton pump inhibitor treatments. Moreover, given the role of Tregs in the immune evasion strategies for some specific pathogens, Tregs depletion has been shown to elicit aggravated gastric mucosal inflammation and bacterial clearance in $H$. pylori-infected mice in vivo ${ }^{103,127}$.

In addition, $H$. pylori eradication therapy may trigger the onset of IBD. However, the evidence supporting this hypothesis is limited and inconclusive, because limited supportive data are available ${ }^{128}$. One case report from Jovanovic et al. ${ }^{129}$ examined one 28-yearold male patient who received 2 weeks of eradication therapy for ulcer-like dyspepsia symptoms. Six months after therapy, he experienced crampy abdominal pain, mild periodical fever, and watery diarrhea, and an endoscopic examination revealed segmental stenotic and Crohn's-like lesions in the upper portion of the small intestines. In addition, Tursi ${ }^{130}$ reported two severe cases of CD (one in the terminal ileitis and one in the cecum and ascending colon) with multiple ulcers and full-thickness lymphoid infiltrates after $H$. pylori eradication therapy. The authors hypothesized that the breakdown of the equilibrium between the Th1 and Th2 responses and subsequent Th1 polarization might favor the onset of $\mathrm{CD}$ in some genetically susceptible individuals. However, in a small-sample H. pylori eradication cohort study ${ }^{131}$ of six patients with quiescent $\mathrm{CD}$, statistically significantly differences in the CDAI (CD activity index), CRP (C-Reactive protein), and fecal calprotectin levels were not observed after $H$. pylori eradication. Further studies are urgently needed to reveal the relationship between $H$. pylori eradication and IBD onset or progression.

\section{Perspectives}

Almost all patients with $H$. pylori infection exhibit chronic inflammation in the gastric mucosa, causing $H$. pylori to be defined as an infectious pathogen according to Koch's law. As H. pylori-induced chronic atrophic gastritis is a crucial risk factor for gastric cancer, the Kyoto global consensus suggests that all $H$. pylori-infected individuals should be treated with eradication unless they present with contraindications to this treatment. Overall, eradication of $H$. pylori has not been confirmed by China's national guidelines, considering the high infection rate and large quantities of antibiotics administered. In fact, the overall effects besides increased gastric cancer risk were largely ignored by the epidemiologists dedicated in H. pylori control. During the long co-evolutionary process with humans, $H$. pylori developed an immune tolerance property that favors its persistent mucosal colonization and simultaneously regulates systematic immune homeostasis by inducing tolerogenic DCs and immunosuppressive Tregs. Thus, the eradication of $H$. pylori with antibiotics not only largely influences the homeostasis of gut microbes but also has an indirect but profound effect on immune homeostasis and may lead to various autoimmune and allergic diseases, such as IBD and asthma. Just as we could not evaluate the gastric cancer risk in $H$. pylori-infected individuals accurately, we also could not perfectly evaluate the risk of IBD after $H$. pylori eradication, especially for IBD susceptible gene carriers. In conclusion, the immune tolerance property of $H$. pylori 
should be thoroughly considered when designing optimized and individualized treatments for $H$. pylori-infected patients.

\section{Acknowledgements}

This work was completely supported by grants from the National Natural Science Foundation of China (81570507 and 81702314) and Funding Program for Excellent Talents of Beijing (2017000021469G212).

\section{Conflict of interest}

The authors declare that they have no conflict of interest.

\section{Publisher's note}

Springer Nature remains neutral with regard to jurisdictional claims in published maps and institutional affiliations.

Received: 27 March 2018 Revised: 22 August 2018 Accepted: 22 August 2018

Published online: 20 September 2018

\section{References}

1. Baumgart, D. C. \& Sandborn, W. J. Crohn's disease. Lancet380, 1590-1605 (2012).

2. Ordã, sl., Eckmann, L., Talamini, M., Baumgart, D. C. \& Sandborn, W. J. Ulcerative colitis. Lancet 380, 1606-1619 (2012).

3. Manichanh, C., Borruel, N., Casellas, F. \& Guarner, F. The gut microbiota in IBD. Nat. Rev. Gastroenterol. Hepatol. 9, 599-608 (2012).

4. Jostins, L. et al. Host-microbe interactions have shaped the genetic architecture of inflammatory bowel disease. Nature 491, 119-124 (2012).

5. Fujino, S. et al. Increased expression of interleukin 17 in inflammatory bowel disease. Gut 52, 65 (2003).

6. Hue, S. et al. Interleukin-23 drives innate and T cell-mediated intestinal inflammation. J. Exp. Med. 203, 2473-2483 (2006).

7. First, G. et al. Differential regulation of interleukin-17 and interferon-y production in inflammatory bowel disease. Dig. Liver Dis. 58, 1629 (2009).

8. Song, Y. D. \& Zhong, Y. Q. Relationship between autophagy abnormalitis and pathogenesis of inflammatory bowel disease. Chin. J. Gastroenterol. 22, 304-307 (2017).

9. Thia, K. T. et al. An update on the epidemiology of inflammatory bowel disease in Asia. Am. J. Gastroenterol. 103, 3167-3182 (2008).

10. Seksik, P. Gut microbiota and IBD. Gastroenterol. Clin. Biol. 34, S44 (2010). Suppl 1.

11. Johnson, L. D. et al. A prospective study of the epidemiology of colitis and colon cancer in cotton-top tamarins (Saguinus oedipa). Gastroenterology $\mathbf{1 1 0}$ 102-115 (1996).

12. Sellon, R. K. et al. Resident enteric bacteria are necessary for development of spontaneous colitis and immune system activation in interleukin-l0-deficient mice. Infect. Immun. 66, 5224 (1998).

13. Baumgart, D. C. et al. IBD around the world: comparing the epidemiology, diagnosis, and treatment: proceedings of the World Digestive Health Day 2010-Inflammatory Bowel Disease Task Force meeting. Inflamm. Bowel Dis. 17, 639-644 (2011)

14. Molodecky, N. A. et al. Increasing incidence and prevalence of the inflammatory bowel diseases with time, based on systematic review. Gastroenterology 142, 46-54 (2012).

15. Loftus, E. V. Clinical epidemiology of inflammatory bowel disease: incidence, prevalence, and environmental influences. Gastroenterology 126, 1504-1517 (2004).

16. Xavier, R. J. \& Podolsky, D. K. Unravelling the pathogenesis of inflammatory bowel disease. Nature 448, 427 (2007).

17. Zhao, J. et al. First prospective, population-based inflammatory bowel disease incidence study in mainland of China: the emergence of "western" disease. Inflamm. Bowel Dis. 142, 1839-1845 (2013).

18. Kaplan, G. G. \& Ng, S. C. Globalisation of inflammatory bowel disease: perspectives from the evolution of inflammatory bowel disease in the UK and China. Lancet Gastroenterol. Hepatol. 1, 307 (2016).
19. Ng, S. C. et al. Incidence and phenotype of inflammatory bowel disease based on results from the Asiapacific Crohn's and colitis epidemiology study. Gastroenterology 145, E2 (2013).

20. Ye L., Cao Q., Cheng J. Review of inflammatory bowel disease in China. Sci. World J. 2013, 296470 (2013).

21. Dunn, B. E., Cohen, H. \& Blaser, M. J. Helicobacter pylori. Clin. Microbiol. Rev. 10 720-741 (1997).

22. Warren J. R., Marshall B. Unidentified cured bacilli on gastric epithelium in active chronic gastritis. Lancet 1, 1273-1275 (1983).

23. Dooley, C. P. et al. Prevalence of Helicobacter pylori infection and histologic gastritis in asymptomatic persons. New Engl. J. Med. 321, 1562-1566 (1989).

24. Marshall, B. J. \& Warren, J. R. Unidentified curved bacilli in the stomach of patients with gastritis and peptic ulceration. Lancet 1, 1311 (1984).

25. Sugano, K. et al. Kyoto global consensus report on Helicobacter pylori gastritis. Gut 64, 1353-1367 (2015).

26. Parsonnet, J. et al. Helicobacter pylori infection and the risk of gastric carcinoma. N. Engl. J. Med. 325, 1127-1131 (1991).

27. Parsonnet, J. et al. Helicobacter pylori infection and gastric lymphoma. N. Engl. J. Med. 330, 1267-1271 (1994).

28. Kusters, J. G., van Vliet, A. H. \& Kuipers, E. J. Pathogenesis of Helicobacter pylori infection. Clin. Microbiol. Rev. 19, 449-490 (2006).

29. Singh, K. \& Ghoshal, U. C. Causal role of Helicobacter pylori infection in gastric cancer: an Asian enigma. World J. Gastroenterol. 12, 1346-1351 (2006).

30. Arnold, I. C., Iris, H. \& Anne, M. The immunomodulatory properties of Helicobacter pylori confer protection against allergic and chronic inflammatory disorders. Front. Cell. Infect. Microbiol. 2, 10 (2012).

31. Atherton J. C., Blaser M. J. Helicobacter pylori infections. In: Harrison's Principles of Internal Medicine 16th ed, pp 88628 (McGraw-Hill, NewYork: 1998, 2005).

32. Zhang, L., Day, A., McKenzie, G. \& Mitchell, H. Nongastric Helicobacter species detected in the intestinal tract of children. J. Clin. Microbiol. 44, 2276-2279 (2006).

33. Oliveira, A. G. et al. Isolation of Helicobacter pylori from the intestinal mucosa of patients with Crohn's disease. Helicobacter 11, 2-9 (2006).

34. Streutker, C. J., Bernstein, C. N., Chan, V. L., Riddell, R. H. \& Croitoru, K. Detection of species-specific Helicobacter ribosomal DNA in intestinal biopsy samples from a population-based cohort of patients with ulcerative colitis. J. Clin. Microbiol. 42, 660-664 (2004).

35. Man, S. M., Zhang, L., Day, A. S., Leach, S. \& Mitchell, H. Detection of enterohepatic and gastric Helicobacter species in fecal specimens of children with Crohn's disease. Helicobacter 13, 234-238 (2008).

36. Yu, Q. et al. Enterohepatic Helicobacter species as a potential causative factor in inflammatory bowel disease: a meta-analysis. Medicine $\mathbf{9 4}$, e1773 (2015).

37. Hansen, R., Thomson, J. M., Fox, J. G., El-Omar, E. M. \& Hold, G. L. Could Helicobacter organisms cause inflammatory bowel disease? Fems Immunol. Med. Microbiol. 61, 1-14 (2011).

38. Kullberg, M. C. et al. Helicobacter hepaticus triggers colitis in specificpathogen-free interleukin-10 (IL-10)-deficient mice through an IL-12 and gamma interferon dependent mechanism. Infect. Immun. 66, 5157-5166 (1998)

39. Kullberg, M. C. et al. IL-23 plays a key role in Helicobacter hepaticus-induced T cell-dependent colitis. J. Exp. Med. 203, 2485-2494 (2006).

40. Chow, J. \& Mazmanian, S. K. A pathobiont of the microbiota balances host colonization and intestinal inflammation. Cell. Host. Microbe 7, 265-276 (2010).

41. Young, V. B. \& Schauer, D. B. Cytolethal distending toxin: a bacterial toxin which disrupts the eukaryotic cell cycle. Chem. Res. Toxicol. 13, 936-939 (2000).

42. Kuehl, C. J., Wood, H. D., Marsh, T. L., Schmidt, T. M. \& Young, V. B. Colonization of the cecal mucosa by Helicobacter hepaticus impacts the diversity of the indigenous microbiota. Infect. Immun. 73, 6952-6961 (2005).

43. Whary, M. T. et al. Rapid onset of ulcerative typhlocolitis in B6.129P2IL10tm1Cgn (IL-10-/-) mice infected with Helicobacter trogontum is associated with decreased colonization by altered Schaedler's flora. Infect. Immun. 74, 6615 (2006)

44. Halme, L., Rautelin, H., Leidenius, M. \& Kosunen, T. U. Inverse correlation between Helicobacter pylori infection and inflammatory bowel disease. J. Clin. Pathol. 49, 65-67 (1996).

45. Pearce, C. B., Duncan, H. D., Timmis, L. \& Green, J. R. Assessment of the prevalence of infection with Helicobacter pylori in patients with inflammatory bowel disease. Gastroenterology 118, 439-443 (2000). 
46. Sukerek, H. H., Thomas, R. L. \& Tolia, V. K. Incidence of Helicobacter pylori, infection in pediatric inflammatory bowel disease patients. Am. J. Gastroenterol. 96, S310-S310 (2001).

47. Väre, P. O. et al. Seroprevalence of Helicobacter pylori infection in inflammatory bowel disease: is Helicobacter pylori infection a protective factor? Scand. J. Gastroenterol. 36, 1295-1300 (2001).

48. Matsumura, M. et al. Prevalence of Helicobacter pylori infection and correlation between severity of upper gastrointestinal lesions and $H$. pylori infection in Japanese patients with Crohn's disease. J. Gastroenterol. 36, 740-747 (2001).

49. Feeney, M. A. et al. A case-control study of childhood environmental risk factors for the development of inflammatory bowel disease. Eur. J. Gastroenterol. Hepatol. 14, 529-534 (2002).

50. Parlak, E., Ulker, A., Dişibeyaz, S., Alkim, C. \& Dağli, U. There is no significant increase in the incidence of Helicobacter pylori infection in patients with inflammatory bowel disease in Turkey. J. Clin. Gastroenterol. 33, 87-88 (2001).

51. Prónai, L., Schandl, L., Orosz, Z., Magyar, P. \& Tulassay, Z. Lower prevalence of Helicobacter pylori infection in patients with inflammatory bowel disease but not with chronic obstructive pulmonary disease-antibiotic use in the history does not play a significant role. Helicobacter 9, 278-283 (2004).

52. Stadek, M. et al. The low prevalence of Helicobacter pylori gastritis in newly diagnosed inflammatory bowel disease children and adolescent. Przegl. Lek. 64, 65-67 (2007).

53. Song, M. J. et al. [The prevalence of Helicobacter pylori infection in Korean patients with inflammatory bowel disease, a multicenter study]. Korean J. Gastroenterol. Taehan Sohwagi Hakhoe Chi 53, 341-347 (2009).

54. Pang, Z., Li, M. F., Zhao, H., Zhou, C. L. \& Shen, B. W. Low prevalence of Helicobacter pylori infection in Chinese Han patients with inflammatory bowel disease[J]. World J. Gastroenterol. 17, 3661-3665 (2009).

55. XQ, L. I., Wu, Y. S., Pan, L. J. \& Tao, Z. Q. Correlation study between Helicobacter pylori and ulcerative colitis. Mod. Med. J. 38, 647-648 (2010). De.

56. Pellicano, R. et al. Prevalence of Helicobacter pylori infection in patients with inflammatory bowel disease: pilot study. Rev. Esp. Enferm. Dig. 102, 675 (2010).

57. Zhang, S. et al. Role of Helicobacter species in Chinese patients with inflammatory bowel disease[J]. J. Clin. Microbiol. 49, 1987 (2011).

58. Sonnenberg, A. \& Genta, R. M. Low prevalence of Helicobacter pylori, infection among patients with inflammatory bowel disease. Aliment. Pharmacol. Ther. 35, 469-476 (2012).

59. Xiang, Z. et al. Helicobacter pylori and Crohn's disease: a retrospective singlecenter study from China. World J. Gastroenterol. 19, 4576-4581 (2013).

60. Jin, X., Chen, Y. P., Chen, S. H. \& Xiang, Z. Association between Helicobacter pylori infection and ulcerative colitis-a case control study from China. Int. J. Med. Sci. 10, 1479-1484 (2013).

61. Xin, Y., Jiang, Y., Zhang, Z. G., Oi, F. X. \& Li, M. Relationship between Helicobacter pylori infection and inflammatory bowel disease. Med. Inf. 11, 383-384 (2013).

62. Ali, A., Kozcka, C., Masood, U., Alam, A. \& Lawlor, G. Low prevalence of Helicobacter pylori infection in IBD patients from a predominantly African/ Caribbean urban center. Inflamm. Bowel Dis. 19, S55-S56 (2013).

63. Roka, K. et al. The prevalence of Helicobacter pylori gastritis in newly diagnosed children with inflammatory bowel disease. Helicobacter 19, 400-405 (2014).

64. Ma T. H., Yang X., Xie R. H., Yan W. Clinical analysis of Helicobacter pylori infection in ulcerative colitis patients[J]. J. Shanxi Med. Univ. 47(1), 68-70, (2016)

65. Shi, T. T., Zhu, L. \& Wang, Y. D. Helicobacter pylori infection in patients with inflammatory bowel diseases. China J. Mod. Med. 27, 101-103 (2017).

66. Zhou, L. Y. et al. Relationship between Helicobacter pylori infection and ulcerative colitis. Chin. J. Clin. Res. 30, 447-450 (2017).

67. Luther, J., Dave, M., Higgins, P. D. \& Kao, J. Y. Association between Helicobacter pylori infection and inflammatory bowel disease: a meta-analysis and systematic review of the literature. Inflamm. Bowel Dis. 16, 1077-1084 (2010)

68. Rokkas, T, Gisbert, J. P. Niv, Y. \& O'Morain, C. The association between Helicobacter pylori infection and inflammatory bowel disease based on metaanalysis. United European Gastroenterology. Journal 3, 539-550 (2015).

69. Wu, X. W., Ji, H. Z., Yang, M. F., Wu, L. \& Wang, F. Y. Helicobacter pylori infection and inflammatory bowel disease in Asians: a meta-analysis. World J. Gastroenterol. 21, 4750-4756 (2015).
70. Elomar, E. et al. Low prevalence of Helicobacter pylori in inflammatory bowel disease: association with sulphasalazine. Gut 35, 1385-1388 (1994).

71. Parente, F. et al. Prevalence of Helicobacter pylori infection and related upper gastrointestinal lesions in patients with inflammatory bowel diseases. A cross-sectional study with matching. Scand. J. Gastroenterol. 32, 1140-1146 (1997).

72. Piodi, L. P. et al. Possible protective effect of 5-aminosalicylic acid on Helicobacter pylori infection in patients with inflammatory bowel disease. J. Clin. Gastroenterol. 36, 22-25 (2003).

73. Mantzaris, G. J. et al. Low prevalence of Helicobacter pylori in inflammatory bowel disease: association with sulfasalazine. Am. J. Gastroenterol. 90, 1900 (1995).

74. Castañorodríguez, N. et al. Dual role of Helicobacter and Campylobacter species in IBD: a systematic review and meta-analysis. Gut 66, 235 (2017).

75. Guslandi, M., Fanti, L. \& Testoni, P. A. Helicobacter pylori seroprevalence in Crohn's disease: lack of influence by pharmacological treatment. HepatoGastroenterol. 49, 1296 (2002).

76. Yang, F. et al. Expression of DC-SIGN on intestinal epithelial cells and it's immunity regulation in Helicobacter pylori infection of mouse inflammatory bowel disease. Current. Immunology 1, 23-29 (2013).

77. Higgins, P. D. et al. Prior Helicobacter pylori infection ameliorates Salmonella typhimurium-induced colitis: mucosal crosstalk between stomach and dista intestine. Inflamm. Bowel Dis. 17, 1398-1408 (2011)

78. Kao, J. Y. et al. Helicobacter pylori immune escape is mediated by dendritic cell-induced Treg skewing and Th17 suppression in mice. Gastroenterology 138, 1046-1054 (2010)

79. Leonard, F., Collnot, E. M. \& Lehr, C. M. A three-dimensional coculture of enterocytes, monocytes and dendritic cells to model inflamed intestinal mucosa in vitro. Mol. Pharm. 7, 2103-2119 (2010).

80. Oertli, M. et al. DC-derived IL-18 drives Treg differentiation, murine Helicobacter pylori-specific immune tolerance, and asthma protection. J. Clin. Invest. 122, 1082-1096 (2012).

81. Veldhoen, M., Hocking, R. J., Atkins, C. J., Locksley, R. M. \& Stockinger, B. TGFbeta in the context of an inflammatory cytokine milieu supports de novo differentiation of IL-17-producing T cells. Immunity 24, 179-189 (2006).

82. Korn, $\mathrm{T}$. et al. IL-21 initiates an alternative pathway to induce proinflammatory $T(H) 17$ cells. Nature 448, 484-487 (2007).

83. Ouyang, W., Kolls, J. K. \& Zheng, Y. The biological functions of T helper 17 cell effector cytokines in inflammation. Immunity 28, 454 (2008).

84. Ahern, P. P. et al. Interleukin-23 drives intestinal inflammation through direct activity on T cells. Immunity 33, 279-288 (2010).

85. Muotiala, A., Helander, I. M., Pyhälä, L., Kosunen, T. U. \& Moran, A. P. Low biological activity of Helicobacter pylori lipopolysaccharide. Infect. Immun. $\mathbf{6 0}$ 1714-1716 (1992).

86. Gewirtz, A. T. et al. Helicobacter pylori flagellin evades toll-like receptor 5mediated innate immunity. J. Infect. Dis. 189, 1914-1920 (2004).

87. Wang, Y. H., Gorvel, J. P., Chu, Y. T., Wu, J. J. \& Lei, H. Y. Helicobacter pylori impairs murine dendritic cell responses to infection. PLOS ONE 5, e10844 (2010)

88. Reuter, S., Oertli, M., Engler, D., Maxeiner, J., Buhl, R., \& Muller, A., et al. DC mediated asthma protection by Helicobacter Pylori is dependent on specific virulence factors.Am J Respir Crit Care Med. 187, 4144 (2013).

89. Lord, A. R. et al. Protective effects offor IBD are related to thepositive strain Gut 67, 393-394 (2018)

90. Oertli, M. et al. Helicobacter pylori y-glutamyl transpeptidase and vacuolating cytotoxin promote gastric persistence and immune tolerance. Proc. . Nat/ Acad. Sci. USA. 110, 3047-3052 (2013)

91. Engler, D. B. et al. Effective treatment of allergic airway inflammation with Helicobacter pylori immunomodulators requires BATF3-dependent dendritic cells and IL-10. Proc. Natl Acad. Sci. USA. 111, 11810-11815 (2014)

92. Sakaguchi, S. Naturally arising Foxp3-expressing CD25+CD4+regulatory $\mathrm{T}$ cells in immunological tolerance to self and non-self. Nat. Immunol. $\mathbf{6}$ 345-352 (2005).

93. Park, M. K. et al. Amelioration of autoimmune arthritis by adoptive transfer of Foxp3-expressing regulatory B cells is associated with the Treg/Th17 cell balance. J. Transl. Med. 14, 191 (2016).

94. Lahl, K. 1 et al. Selective depletion of Foxp3 + regulatory T cells induces a scurfy-like disease. J. Exp. Med. 204, 57-63 (2007)

95. Baru, A. M. et al. Correction: selective depletion of Foxp3(+) Treg during sensitization phase aggravates experimental allergic airway inflammation. Eur. J. Immunol. 40, 2655-2655 (2010). 
96. Klingenberg, R. 1 et al. Depletion of FOXP3+regulatory T cells promotes hypercholesterolemia and atherosclerosis. J. Clin. Investig. 123, 1323-1334 (2013).

97. Kim, J. et al. Cutting edge: depletion of Foxp3+cells leads to induction of autoimmunity by specific ablation of regulatory $T$ cells in genetically targeted mice. J. Immunol. 183, 7631 (2009).

98. Du, Y., Chen, X., Lin, X. Q., Wu, W. \& Huang, Z. M. Tumor-derived CD4+CD25 +Tregs inhibit the maturation and antigen-presenting function of dendritic cells. Asian Pac. J. Cancer Prev. 16, 2665-2669 (2015).

99. Sayi, A. et al. TLR-2-activated B cells suppress Helicobacter-induced preneoplastic gastric immunopathology by inducing $T$ regulatory-1 cells. J. Immunol. 186, 878-890 (2011).

100. Lundgren, A. et al. Mucosal FOXP3-expressing CD4+CD25high regulatory $\mathrm{T}$ cells in Helicobacter pylori-infected patients. Infect. Immun. 73, 523-531 (2005)

101. Arnold, I. C. et al. Helicobacter pylori infection prevents allergic asthma in mouse models through the induction of regulatory T cell. J. Clin. Investig. 121, 3088-3093 (2011).

102. Driel, I. R. V. \& Ang, D. K. Role of regulatory T cells in gastrointestinal inflammatory disease. J. Gastroenterol. Hepatol. 23, 171-177 (2008).

103. Izcue, A., Coombes, J. L. \& Powrie, F. Regulatory T cells suppress systemic and mucosal immune activation to control intestinal inflammation. Immunol. Rev. 212, 256-271 (2006).

104. Mottet, C., Uhlig, H. H. \& Powrie, F. Cutting edge: cure of colitis by CD4 +CD25+regulatory T cells. J. Immunol. 170, 3939-3943 (2003).

105. Luther, J. et al. Helicobacter pylori DNA decreases proinflammatory cytokine production by dendritic cells and attenuates dextran sodium sulphateinduced colitis. Gut 60, 1479 (2011).

106. Onishi, Y., Fehervari, Z., Yamaguchi, T. \& Sakaguchi, S. Foxp3+natural regulatory $T$ cells preferentially form aggregates on dendritic cells in vitro and actively inhibit their maturation. Proc. Natl Acad. Sci. USA. 105, 10113-11011 (2008)

107. Leach, M. W., Davidson, N. J., Fort, M. M., Powrie, F. \& Rennick, D. M. The role of IL-10 in inflammatory bowel disease: "of mice and men". Toxicol. Pathol. 27, 123-133 (1999).

108. Ohtsuka, Y. \& Sanderson, I. R. Transforming growth factor-beta: an important cytokine in the mucosal immune response. Curr. Opin. Gastroenterol. 16 541-545 (2000).

109. Zou, L. et al. The association between three promoter polymorphisms of IL10 and inflammatory bowel diseases (IBD): a meta-analysis. Autoimmunity 47, 27-39 (2014)

110. Begue, B. et al. Defective IL10 signaling defining a subgroup of patients with inflammatory bowel disease. Am. J. Gastroenterol. 106, 1544 (2011).

111. Yao X. C., Wang J. Expression level of TREG, TGF- $\beta$ and IL-10 in IBD patients and their clinical value. J. Shandong Med. Coll. 39, 107-110 (2017).

112. Powrie, F., Carlino, J., Leach, M. W., Mauze, S. \& Coffman, R. L. A critical role for transforming growth factor- $\beta$ but not interleukin 4 in the suppression of $T$ helper type 1-mediated colitis by CD45RB low CD4+T cells. J. Exp. Med. 183 2669-2674 (1996).

113. Neurath, M. F. et al. Experimental granulomatous colitis in mice is abrogated by induction of TGF- $\beta$-mediated oral tolerance. J. Exp. Med 183, 2605-2616 (1996).
114. Ruiz, P. A., Shkoda, A., Kim, S. C., Sartor, R. B. \& Haller, D. IL-10 gene deficient mice lack TGF-beta/Smad signaling and fail to inhibit proinflammatory gene expression in intestinal epithelial cells after the colonization with colitogenic Enterococcus faecalis. J. Immunol. 174, 2990-2999 (2005).

115. Liu, X., Zhang, Y., Yu, Y., Yang, X. \& Cao, X. Socs3 promotes tlr4 response in macrophages by feedback inhibiting tgf-beta1/smad3 signaling. Mol. Immunol. 45, 1405-1413 (2008).

116. Engler, D. B. et al. Helicobacter pylori-specific protection against inflammatory bowel disease requires the nlrp3 inflammasome and il-18. Inflamm. Bowel Dis. 21, 854-861 (2015).

117. Kim, D. J., Park, J. H., Franchi, L., Backert, S. \& Núñez, G. The cag pathogenicity island and cooperative interaction between $\operatorname{tr} 2 / \operatorname{nod} 2$ and nlrp3 regulate it$1 \beta$ production in Helicobacter pylori-infected dendritic cells. Eur. J. Immunol. 43, 2650-2658 (2014).

118. Hitzler, I. et al. Caspase-1 has both proinflammatory and regulatory properties in Helicobacter infections, which are differentially mediated by its substrates IL-1 beta and IL-18. J. Immunol. 188, 3594-3602 (2012).

119. Coccia, $M$. et al. IL-1 $\beta$ mediates chronic intestinal inflammation by promoting the accumulation of IL-17A secreting innate lymphoid cells and CD4+Th17 cells. J. Exp. Med. 209, 1595-1609 (2012)

120. Sharif, O., Bolshakov, V. N., Raines, S., Newham, P. \& Perkins, N. D. Transcriptional profiling of the LPS induced NF-KB response in macrophages. Bmc. Immunol. 8, 1 (2007).

121. Kwon, K. H., Murakami, A., Hayashi, R. \& Ohigashi, H. Interleukin-1 beta targets interleukin-6 in progressing dextran sulfate sodium-induced experimental colitis. Biochem. Biophys. Res. Commun. 337, 647-654 (2005).

122. Nan, L., Xu, T., Lingfen, X. \& Mei, S. The effects of $I L-1$ beta on intestinal epithelial barrier permeability. Int. J. Pediatr. 43, 5 (2016).

123. Serrano, C. et al. Downregulated Th17 responses are associated with reduced gastritis in Helicobacter pylori infected children. Mucosal. Immunology 6, 950 (2013).

124. Harris, P. R. et al. Helicobacter pylori gastritis in children is associated with a regulatory T-cell response. Gastroenterology 134, 491-499 (2008).

125. Engler D., Muller A. Beneficial effects of early childhood Helicobacter pylori infection on the development of allergic and chronic inflammatory disorders. Allergy. 67, 93 (2012).

126. Wang, G. et al. "Default" generation of neonatal regulatory T cells. J. Immunol. 185, 71-78 (2010)

127. Rad, R. et al. CD25+/Foxp3+T cells regulate gastric inflammation and Helicobacter pylori colonization in vivo. Gastroenterology 131, 525 (2006).

128. Murad, H. A. Does Helicobacter pylori eradication therapy trigger or protect against Crohn's disease? Acta Gastroenterol. Belg. 79, 349 (2016).

129. Jovanovic, I. R et al. Clinical onset of the Crohn's disease after eradication therapy of Helicobacter pylori infection. Does Helicobacter pylori infection interact with natural history of inflammatory bowel diseases? Medical Science Monitor International Medical. J. Exp. Clin. Res. 7, 137 (2001).

130. Tursi, A. Onset of Crohn's disease after Helicobacter pylori eradication. Inflamm. Bowel Dis. 12, 1008-1009 (2006).

131. Lahat, A. et al. Helicobacter pylori prevalence and clinical significance in patients with quiescent Crohn's disease. BMC Gastroenterol. 17, 27 (2017). 